\title{
Motivation to change and posttreatment temptation to drink: a multicenter study among alcohol-dependent patients
}

This article was published in the following Dove Press journal:

Neuropsychiatric Disease and Treatment

3 October 2017

Number of times this article has been viewed

\author{
Elena Fiabane ${ }^{1,2}$ \\ Marcella Ottonello1,3 \\ Valeria Zavan ${ }^{4}$ \\ Caterina Pistarini' \\ Ines Giorgi ${ }^{5}$
}

'Department of Physical and Rehabilitation Medicine, ICS Maugeri Spa SB, Institute of Genoa Nervi, Genoa, ${ }^{2}$ Department of Brain and Behavioral Sciences, University of Pavia, Pavia, ${ }^{3}$ Department of Medicine, PhD Program in Advance Sciences and Technologies in Rehabilitation Medicine and Sport, Università di Tor Vergata, Rome, ${ }^{4}$ Alcohol Unit, Addiction Department, Alessandria, ${ }^{5}$ Psychology Unit, ICS Maugeri Spa SB, Scientific Institute of Pavia, Pavia, Italy

Correspondence: Elena Fiabane Department of Physical and Rehabilitation Medicine, ICS Maugeri Spa SB, Institute of Genoa Nervi, Via Missolungi 14, Nervi, Genoa 16167, Italy Tel +3901030791252

Fax +39010 30791269

Email elenamaria.fiabane@unipv.it
Background: An inpatient 28-day rehabilitation program for alcohol dependence is focused on detoxification, enhancing motivation to change, and coping strategies for the management of emotional distress and temptation to drink. The aims of the present study were to investigate 1) changes over time in motivation to change, anxiety, and depression in individuals undergoing residential rehabilitation treatment for alcohol addiction, and 2) which pretreatment factors are associated with posttreatment temptation to drink.

Methods: We assessed 432 patients diagnosed with alcohol dependence based on the Diagnostic and Statistical Manual of Mental Disorders (Fourth Edition, Text Revision) consecutively recruited from seven residential rehabilitation centers in Northern Italy. Patients were assessed by means of self-report questionnaires at the beginning of rehabilitation (pretreatment) and before their hospital discharge (posttreatment) regarding motivation to change and self-efficacy (motivation assessment of change - alcoholism version), readiness to change and temptation to drink (visual analog scales), and depression and anxiety (cognitive behavioral assessment - outcome evaluation).

Results: Results showed an overall improvement in motivation to change and a significant reduction over time in depression and anxiety levels for the total sample, particularly patients with low baseline level of temptation to drink. Multivariate linear regression analysis revealed that significant predictors of posttreatment temptation to drink were polysubstance dependence $(p<0.001)$, readiness to change ( $p=0.01)$, and self-efficacy $(p=0.05)$.

Conclusion: Inpatients' rehabilitation for alcohol dependence is associated with an overall improvement in motivation to change and a significant reduction of psychological distress. Treatments for alcohol-dependent patients should focus on motivation to change, negative moods, and relapse prevention strategies for the management of craving.

Keywords: addiction, alcohol, motivation to change, rehabilitation, craving

\section{Introduction}

Alcohol consumption is a major public health issue worldwide and is responsible for $5.9 \%$ of all deaths. Scientific studies conducted by the World Health Organization underlined that $5.1 \%$ of the global burden of disease and injury is attributable to alcohol. ${ }^{1}$

Alcohol is associated with an increased risk of developing health problems (liver cirrhosis, some cancers, and cardiovascular diseases) as well as mental and behavioral disorders, including alcohol dependence. A recent meta-analysis demonstrated that alcohol dependence significantly increased the risk of suicidal ideation, suicide attempt, and completed suicide and the authors concluded that it can be considered an important predictor of suicide and a major cause of premature death. ${ }^{2}$ 
Alcohol consumption has negative effects not only on the drinker, but also on their families and the social context, promoting violent behaviors, social isolation, job loss, and accidents at work and on the road. ${ }^{3-5}$

The treatment of alcohol addiction currently involves a wide variety of effective treatment options which can be performed either in an inpatient or outpatient setting, including medications (eg, disulfiram), cognitive behavioral therapy (CBT), motivational enhancement therapy, 12-step facilitation therapy, contingency management, relapse prevention therapy, and family therapy. ${ }^{6-8}$ During the last 20 years, there has been a growth in the number of short-term residential treatment facilities characterized by highly structured and short-duration treatments involving group therapy, individual therapy, and alcoholism educational programs designed to address the specific behavior of drinking. ${ }^{6,9}$ Previous studies showed an overall reduction in anxiety and depression and an improvement in psychological functioning among alcoholics during the first 3 or 4 weeks after detoxification; $6,10,11$ however, some authors found that the late post-detoxification period is at risk for the onset of depression and relapses, and research is needed to investigate factors associated with stabilization of change and abstinence. ${ }^{10}$

In this context, an individual's "readiness" to change their drinking behavior plays an important role in the alcoholism treatment literature. ${ }^{12,13}$ The transtheoretical model of intentional behavior change outlines a series of stages a person goes through when changing their drinking behavior. ${ }^{14}$ These stages of change include precontemplation, contemplation, preparation, action, and maintenance. In the first stage, the individual is not ready to change and does not intend to start with healthy behavior in the near future. In the contemplation stage, the individual begins to recognize the problem with alcohol but takes no action; in the action stage, the individual is ready for action and is making steps to change behavior; in the last stage, maintenance, the individual works to avoid a relapse. ${ }^{14}$

Some studies have demonstrated the importance of motivation for treatment in predicting session, attendance, recovery, and prognosis. ${ }^{15,16}$ For example, a study on inpatient alcohol treatment found that patients whose motivations to enter treatment were related to current external threats (eg, risk of losing their job, driver's license, or spouse) obtained better treatment outcomes. ${ }^{17}$

After detoxification, patients suffering from alcohol addiction show a high relapse risk and craving or intense desire to keep drinking identified as a trigger for relapse. Craving is defined as an intense and urgent subjective experience that prompts individuals to achieve a target, or practice an activity, to reach its desired effects. ${ }^{18}$

It is clear from the literature that actively desiring alcohol during abuse treatment may be a better predictor of relapse than other variables at admission, such as alcohol severity, psychosocial functioning or treatment duration. ${ }^{19,20}$ Previous studies supported the association between the temptation to drink, in particular subjective craving during treatment, and alcohol relapse..$^{21,22}$

Over the last 40 years, a variety of models for conceptualizing craving have been put forward. However, there is currently no scientific consensus on the factors that influence craving and conceptual definitions of craving vary widely; ${ }^{21}$ a biopsychosocial multidimensional addiction model was proposed but key variables for addiction treatment are still unknown. ${ }^{23}$

Negative affectivity has emerged as a prominent cue for craving in clinical studies. ${ }^{24-26}$ Since craving is a strong predictor of relapse after treatment for alcohol dependence, it could be useful to identify at admission to a rehabilitation program which factors affect craving to better orient treatments.

We carried out this retrospective study to explore changes over time in motivation to change, anxiety, and depression in individuals with alcohol dependence undergoing rehabilitation treatment; we hypothesized an improvement of the motivation to change and a decrease of anxiety and depression at the end of the rehabilitation program. The second aim was to investigate which pretreatment factors are associated with posttreatment temptation to drink; we hypothesized that negative affectivity is a significant predictor of temptation to drink. Finally, since temptation to drink is negatively associated with treatment outcomes, we compared the psychological variations over time between patients with low versus medium-high baseline level of temptation to drink.

\section{Methods}

\section{Participants}

This retrospective study was carried out on 649 inpatients consecutively recruited from seven inpatient 28-day residential rehabilitation centers for alcohol addiction in Northern Italy.

Of 649 patients, $170(26.2 \%)$ were excluded from the study or refused to participate and $47(7.2 \%)$ were excluded from the subsequent analyses because of missing values on key variables (for details, see Figure 1). The final study sample consisted of 432 patients diagnosed with alcohol dependence according to the Diagnostic and Statistical 


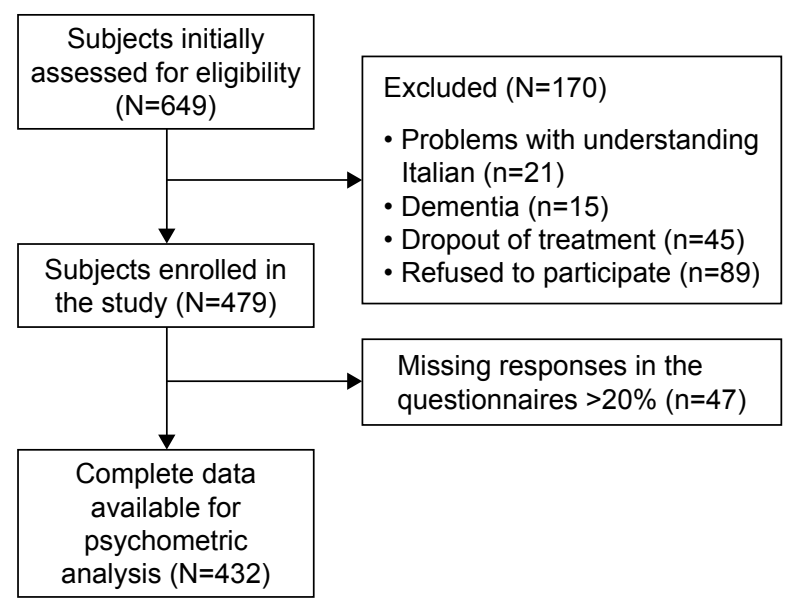

Figure I Flowchart showing the stratification of patients enrolled in the study.

Manual of Mental Disorders, Fourth Edition, Text Revision (DSM-IV-TR). ${ }^{27}$ All participants were informed about the aims of the study and informed consent was obtained.

Patients were assessed by means of self-report questionnaires at the end of their first week of residential treatment after complete detoxification (pretreatment, T1) and before their hospital discharge (posttreatment, T2). The residential rehabilitation program lasted 28 days and was characterized by a high intensity of medical and psychotherapeutic treatment, including CBT group therapy, individual psychological therapy, physical training, health education sessions, and skills training. ${ }^{6}$

All data collected in this study were derived from tools and treatments used in routine clinical practice of the centers involved. This research is a second part of the study previously published in this journal and approved by the institutional review board (Committee of the Care and Research Institute of Tradate, Salvatore Maugeri Foundation, Varese, Italy). ${ }^{6}$

\section{Measures}

\section{Motivation to change and self-efficacy}

These were assessed using the motivation assessment of change - alcoholism version (MAC2-A). ${ }^{28}$ It is a 36 -item self-report measure of motivation for change in individuals with alcohol problems based on the transtheoretical model. ${ }^{14}$ The Likert-type scale ranges from 0 (not at all true) to 6 (completely true) for each item. For this study, we used the total scores for each of the six stages of change (precontemplation, contemplation, determination, action, maintenance, and termination) (18 items) and the self-efficacy subscale ( 6 items). This instrument has demonstrated good reliability and validity in measuring motivation for change in the Italian population. ${ }^{28}$

\section{Readiness to change and temptation to drink}

These were assessed using two 100-point visual analog scales (VASs) included in the MAC2-A questionnaire which used a response format ranging from 0 (not at all) to 100 (extremely). ${ }^{28}$

\section{Depression and anxiety}

These were assessed using two scales from the cognitive behavioral assessment - outcome evaluation questionnaire. ${ }^{29}$ The depression scale (19 items) and the anxiety scale (14 items) were scored on a 5-point Likert-type scale ranging from 1 (nothing) to 5 (a lot). Both scales demonstrated excellent reliability in clinical groups (depression, $\alpha=0.91$; anxiety, $\alpha=0.90) .{ }^{29}$

\section{Statistical analysis}

Descriptive statistics were used to analyze characteristics of the sample (eg, age, sex, education, polysubstance dependence). The temptation to drink scale was dichotomized considering the median of the scores distribution, to obtain two separate groups of patients characterized by low $(\leq 20)$ and medium-high $(>20)$ temptation to drink levels. This method has been widely used in previous scientific studies. ${ }^{30,31}$

Data were separately analyzed for each psychological variable by means of repeated measures analysis of variance (ANOVA), with time (T1, T2) as the within-subject factor and temptation to drink (low vs medium-high) as the between-subject factor. The dependent variables in each analysis were the stages of change, self-efficacy, readiness to change, depression, and anxiety.

Pearson's correlation analysis was performed to examine bivariate correlations among pretreatment factors (eg, depression, readiness to change) and posttreatment temptation to drink. Variables which showed a significant correlation with posttreatment temptation to drink $(p<0.05)$ were included simultaneously in a multivariate regression model to identify baseline predictors of posttreatment temptation to drink. A $p$-value $<0.05$ was considered statistically significant.

All statistical analyses were conducted using SPSS, Windows version 13.0 (SPSS Inc, Chicago, IL, USA).

\section{Results}

Patients were predominantly male (70.8\%) and had a mean age of $46.47 \pm 10.13$ years; $49.3 \%$ were employed and $65.5 \%$ had a low education level. The majority of patients had a history of $>10$ years of alcohol dependence $(55.1 \%)$ and 
Table I Sociodemographic and clinical characteristics of total sample $(n=432)$

\begin{tabular}{|c|c|c|c|}
\hline Variable & $\begin{array}{l}\text { Male }(N=306) \\
n(\%)\end{array}$ & $\begin{array}{l}\text { Female }(\mathrm{N}=\mid 26) \\
\mathrm{n}(\%)\end{array}$ & $\chi^{2}$ or $\mathbf{F}$ \\
\hline \multicolumn{3}{|l|}{ Education } & 4.12 \\
\hline Low & $201(74.4)$ & $69(25.6)$ & \\
\hline Medium & $63(65.6)$ & $33(34.4)$ & \\
\hline High & $34(64.2)$ & $19(35.8)$ & \\
\hline \multicolumn{4}{|l|}{ Missing $=13$} \\
\hline \multicolumn{3}{|l|}{ Occupation } & $18.95^{* * * *}$ \\
\hline Employed & I7I (80.3) & $42(19.7)$ & \\
\hline Not employed & $104(60.1)$ & 69 (39.9) & \\
\hline Retired & $27(69.2)$ & $12(30.8)$ & \\
\hline \multicolumn{4}{|l|}{ Missing $=7$} \\
\hline \multicolumn{3}{|c|}{ Alcohol dependence } & 0.21 \\
\hline$<5$ years & $50(73.5)$ & $18(26.5)$ & \\
\hline $5-10$ years & $64(64.0)$ & $36(36.0)$ & \\
\hline$>10$ years & $174(73.1)$ & $64(26.9)$ & \\
\hline \multicolumn{4}{|l|}{ Missing $=26$} \\
\hline \multicolumn{3}{|c|}{ Polysubstance dependence } & 1.02 \\
\hline No & $210(72.9)$ & $78(27.1)$ & \\
\hline Yes & $92(68.1)$ & 43 (31.9) & \\
\hline \multicolumn{4}{|l|}{ Missing $=9$} \\
\hline Age & & & 0.09 \\
\hline Mean (SD) & $46.38(10.28)$ & 46.7I (9.8I) & \\
\hline
\end{tabular}

Note: $* * * p<0.001$.

Abbreviation: SD, standard deviation.

did not have polysubstance dependence (66.7\%). Employment rates were significantly $(p<0.001)$ higher for males $(80.3 \%)$ than females $(19.7 \%)$. The sociodemographic and clinical characteristics of the total sample are represented in Table 1.
Modifications over time in the stages of change for the total sample are described in Figure 2. Specifically, there was a significant posttreatment decrease in precontemplation $(\mathrm{F}=19.82 ; p<0.001)$ and contemplation mean levels $(\mathrm{F}=6.41$; $p<0.01$ ), and a significant posttreatment increase in action $(\mathrm{F}=39.86 ; p<0.001)$, maintenance $(\mathrm{F}=188.12 ; p<0.001)$, and termination $(\mathrm{F}=161.19 ; p<0.001)$ mean levels compared to pretreatment levels.

ANOVA showed significant main effects of both time and group for the following stages of change: precontemplation (time $\mathrm{F}=19.75 ; p<0.001$; group $\mathrm{F}=5.90 ; p<0.01$ ); action (time $\mathrm{F}=42.02 ; p<0.001$; group $\mathrm{F}=33.41 ; p<0.001$ ); maintenance (time $\mathrm{F}=195.58 ; p<0.001$; group $\mathrm{F}=36.45 ; p<0.01$ ); and termination (time $\mathrm{F}=164.74 ; p<0.001$; group $\mathrm{F}=78.66$; $p<0.001)$; a significant main effect of time was found for contemplation $(\mathrm{F}=6.45 ; p<0.01)$ and significant effect of group was found for determination $(\mathrm{F}=14.06$; $p<0.001$ ).

In relation to self-efficacy, ANOVA showed both a significant main effect of time $(\mathrm{F}=197.33 ; p<0.001)$ and a significant main effect of group $(\mathrm{F}=78.25 ; p<0.001)$.

In relation to readiness to change, ANOVA showed both a significant main effect of time $(\mathrm{F}=7.06 ; p<0.01)$ and a significant main effect of group ( $\mathrm{F}=24.45 ; p<0.001)$.

In relation to depression, ANOVA showed both a significant main effect of time $(\mathrm{F}=485.54 ; p<0.001)$ and a significant main effect of group $(\mathrm{F}=22.65 ; p<0.001)$. Also, anxiety

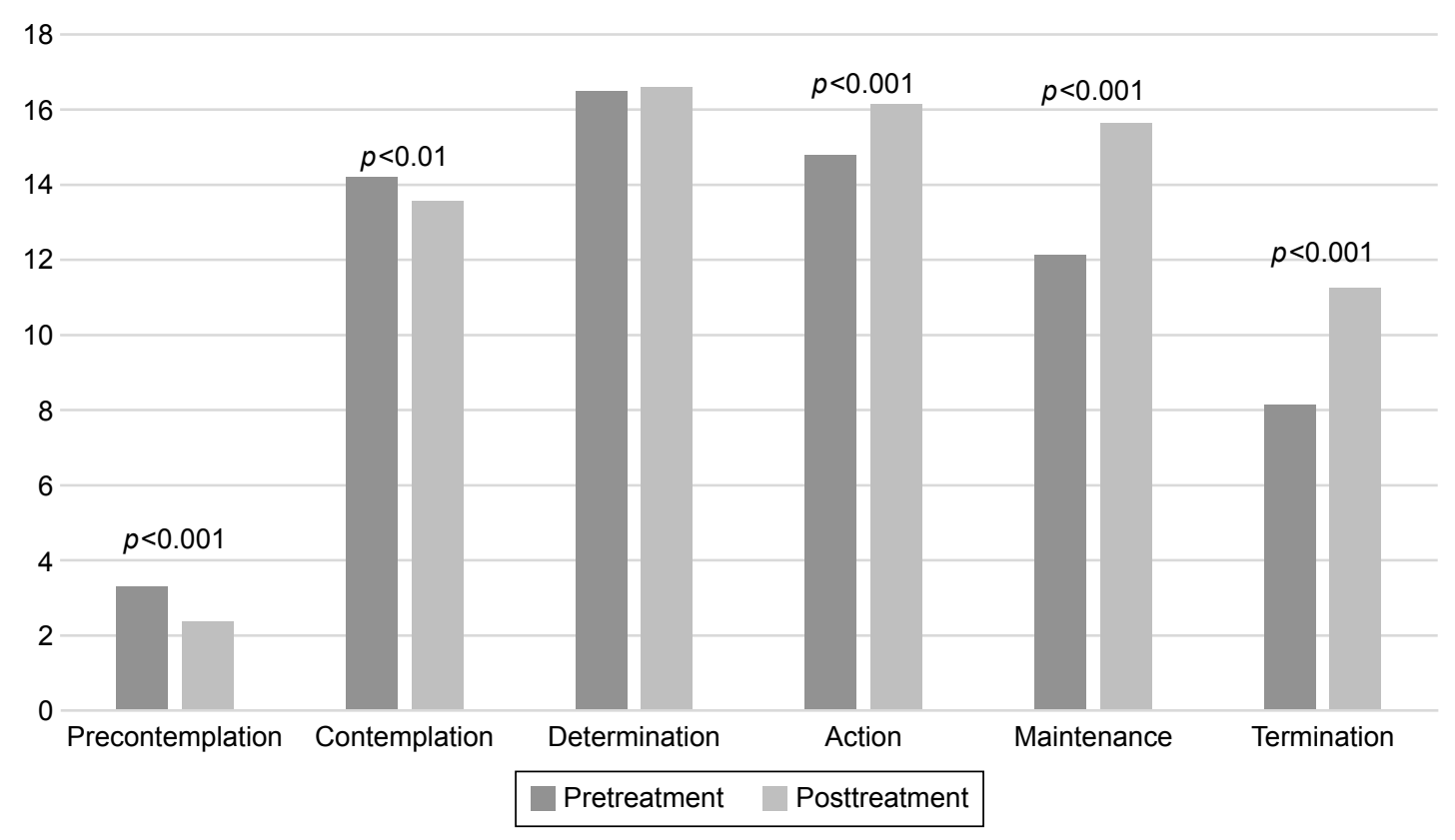

Figure 2 Modifications of stages of change during the rehabilitation program for alcohol addiction.

Notes: Differences in motivation to change (mean scores) between pre- and post-rehabilitation according to the transtheoretical model of stages of change. The area with the highest score was considered as the prevalent stage of change. 
Table 2 Motivation to change and psychological health during a 28-day rehabilitation program: effect of time and temptation to drink

\begin{tabular}{|c|c|c|c|c|c|c|c|c|}
\hline \multirow[t]{3}{*}{ Variable } & \multicolumn{3}{|c|}{ Pretreatment } & \multicolumn{3}{|c|}{ Posttreatment } & \multirow[t]{2}{*}{ Time } & \multirow[t]{2}{*}{ Group $^{a}$} \\
\hline & $\begin{array}{l}\text { No-low } \\
\text { temptation } \\
\text { to drink }\end{array}$ & $\begin{array}{l}\text { Medium-high } \\
\text { temptation } \\
\text { to drink }\end{array}$ & Total & $\begin{array}{l}\text { No-low } \\
\text { temptation } \\
\text { to drink }\end{array}$ & $\begin{array}{l}\text { Medium-high } \\
\text { temptation } \\
\text { to drink }\end{array}$ & Total & & \\
\hline & $M(S D)$ & $M(S D)$ & $M(S D)$ & $M(S D)$ & $M(S D)$ & $M(S D)$ & $\mathbf{F}$ & $\mathbf{F}$ \\
\hline Precontemplation & $2.99(3.89)$ & $3.65(3.77)$ & $3.31(3.85)$ & $2.06(3.06)$ & $2.73(3.21)$ & $2.38(3.15)$ & $19.75^{* * *}$ & $5.90 * *$ \\
\hline Contemplation & $14.34(3.36)$ & $14.04(3.06)$ & $14.19(3.22)$ & |3.8| (5.07) & $13.29(3.98)$ & $13.56(4.57)$ & $6.45^{* *}$ & 1.93 \\
\hline Determination & $17.04(2.15)$ & $15.90(2.56)$ & $16.49(2.42)$ & $16.80(2.74)$ & $16.38(2.4 I)$ & $16.60(2.59)$ & 0.96 & $14.06 * * *$ \\
\hline Action & I5.85 (3.34) & I3.65 (4.54) & I 4.78 (4.II) & $16.52(2.4 I)$ & $15.73(3.10)$ & 16.14 (2.79) & $42.02 * * *$ & $33.41^{* * *}$ \\
\hline Maintenance & I3.47 (4.66) & $10.68(5.30)$ & $12.12(5.17)$ & $16.15(2.87)$ & $15.09(3.43)$ & $15.64(3.19)$ & $195.58 * * *$ & $36.45 * * *$ \\
\hline Termination & $10.21(4.98)$ & $5.94(4.82)$ & $8.14(5.34)$ & $12.74(4.69)$ & $9.66(5.15)$ & $11.25(5.15)$ & $164.74^{* * *}$ & $78.66 * * *$ \\
\hline Self-efficacy & $24.62(7.32)$ & $18.20(7.28)$ & $21.50(7.97)$ & $28.47(6.21)$ & $24.71(7.08)$ & $26.65(6.90)$ & $197.33^{* * * *}$ & $78.25 * * *$ \\
\hline Readiness to change & $84.70(18.88)$ & $76.16(20.82)$ & $80.55(20.28)$ & $86.18(15.69)$ & $79.74(17.26)$ & $83.06(16.76)$ & $7.06 * *$ & $24.45 * * *$ \\
\hline Depression & 27.49 (14.67) & $32.86(13.56)$ & 30.05 (14.39) & $13.69(8.69)$ & $17.96(10.63)$ & I5.73 (9.89) & $485.54 * * *$ & $22.65 * * *$ \\
\hline Anxiety & 23.31 (II.73) & $26.82(10.81)$ & $24.98(11.42)$ & I2.82 (7.90) & I 6.55 (9.7I) & $14.60(9.00)$ & $402.88 * * *$ & $17.76 * * *$ \\
\hline
\end{tabular}

Notes: ${ }^{* *} p<0.01 ; * * *<0.001$. aNo-low temptation to drink versus medium-high temptation to drink. Data presented as mean (SD). Within-subject factor: time (pretreatment, posttreatment); between-subject factor: temptation to drink (low versus medium-high temptation to drink). Dependent factors: six stages of change, self-efficacy, readiness to change, depression, and anxiety.

Abbreviations: $M$, mean; SD, standard deviation.

revealed both a significant main effect of time $(\mathrm{F}=402.88$; $p<0.001)$ and a significant main effect of group $(\mathrm{F}=17.76$; $p<0.001$ ). Results are summarized in Table 2 .

Results of the correlation analysis (Table 3 ) showed that posttreatment temptation to drink had a significantly negative correlation with age $(r=-0.18 ; p<0.001)$, readiness to change $(r=-0.23 ; p<0.001)$, and self-efficacy $(r=-0.32 ; p<0.001)$ and a significantly positive correlation with polysubstance dependence $(r=0.20 ; p<0.001)$, anxiety $(r=0.18 ; p<0.001)$, and depression $(r=0.21 ; p<0.001)$.

Results of the multivariate linear regression analysis are presented in Table 4. Significant predictors of posttreatment temptation to drink were polysubstance dependence $(\beta=0.18$; $p<0.001)$, readiness to change $(\beta=-0.12 ; p=0.01)$, and selfefficacy $(\beta=-0.10 ; p=0.05)$. This model was adjusted for pretreatment temptation to drink level and rehabilitation center, and explained $24.0 \%$ of the total variance.

\section{Discussion}

The first aim of this study was to explore modifications in motivation to change, anxiety, and depression among alcoholdependent patients during their early detoxification period.

Concerning motivation to change, our findings showed an overall significant improvement in motivation to change for the total sample. Residential rehabilitation programs for alcohol disorders are designed to detoxify the individual and to enhance their motivation to change, coping strategies, and interpersonal skills. ${ }^{6}$ Patients moved from the initial stages of change (precontemplation and contemplation), where addicted individuals have little interest in considering the change, to the stages of action, maintenance, and termination where they implement the plan and new behavior becomes normative. $^{12}$

The stages of change have been found to be important predictors of treatment outcomes for many disorders, including

Table 3 Correlations among posttreatment temptation to drink and pretreatment factors

\begin{tabular}{|c|c|c|c|c|c|c|c|c|c|}
\hline Variable & $\mathbf{I}$ & 2 & 3 & 4 & 5 & 6 & 7 & 8 & 9 \\
\hline I. Temptation to drink & I & & & & & & & & \\
\hline 2. Gender ${ }^{\mathrm{a}}$ & 0.04 & 1 & & & & & & & \\
\hline 3. Age & $-0.18^{* *}$ & 0.01 & 1 & & & & & & \\
\hline 4. Polysubstance dependence ${ }^{b}$ & $0.20 * *$ & 0.05 & -0.22 & 1 & & & & & \\
\hline 5. Alcohol dependence (years) & 0.02 & -0.02 & $0.20 * *$ & 0.05 & I & & & & \\
\hline 6. Self-efficacy & $-0.32 * *$ & -0.02 & $0.16 * *$ & -0.02 & 0.03 & I & & & \\
\hline 7. Readiness to change & $-0.23 * *$ & -0.02 & 0.07 & -0.07 & -0.04 & $0.29 * *$ & I & & \\
\hline 8. Depression & $0.21 * *$ & $0.13^{* *}$ & $-0.13^{* *}$ & 0.09 & -0.09 & $-0.37^{* *}$ & $-0.12^{*}$ & I & \\
\hline 9. Anxiety & $0.18^{* *}$ & 0.20 *** & $-0.22 * *$ & $0.13 *$ & -0.08 & -0.3 I** & $-0.11 *$ & $0.83^{* *}$ & I \\
\hline
\end{tabular}

Notes: Data are presented as Pearson's correlation coefficient $(r)$. a $\mathrm{l}=$ male, $2=$ female; ${ }^{\text {b }}=$ no, $2=$ yes. ${ }^{*} p<0.05$; ${ }^{* *} p<0.0 \mathrm{I}$. 
Table 4 Multivariate regression model for predictors of posttreatment temptation to drink*

\begin{tabular}{lllll}
\hline Independent variable & $\mathbf{B}$ & $\boldsymbol{\beta}$ & $\mathbf{T}$ & $\boldsymbol{p}$-value \\
\hline Age & -0.12 & -0.06 & -1.27 & 0.20 \\
Gender & 0.16 & 0.00 & 0.08 & 0.93 \\
Polysubstance dependence & 7.99 & 0.18 & 3.89 & $<0.001$ \\
Anxiety & -0.11 & -0.06 & -0.73 & 0.47 \\
Depression & 0.13 & 0.09 & 1.05 & 0.29 \\
Self-efficacy & -0.26 & -0.10 & -1.93 & 0.05 \\
Readiness to change & -0.12 & -0.12 & -2.46 & 0.01 \\
\hline
\end{tabular}

Notes: *The model is adjusted for pretreatment temptation to drink level and rehabilitation center. $R^{2}=0.24$; adjusted $R^{2}=0.22$. B, partial regression coefficient; $\beta$, standardized partial regression coefficient. Dependent factor, posttreatment temptation to drink. Independent factors: age, $\operatorname{sex}($ male $=I$, female $=2)$, polysubstance dependence ( $n o=I$, yes $=2$ ), pretreatment anxiety score, pretreatment depression score, pretreatment self-efficacy score, pretreatment readiness to change score.

alcohol problems. ${ }^{32}$ For example, a previous study found that posttreatment (and not pretreatment) stage of change was predictive of drinking outcomes at 12-month follow-up. ${ }^{33}$ Additionally, Cadigan et al found that the highest levels of alcohol were associated with moderate levels of readiness to change among college students. ${ }^{34}$

Concerning anxiety and depression, we found a significant reduction in the levels of both during the rehabilitation program. These findings confirm previous research which showed a decrease in psychological distress during the early alcohol detoxification period. ${ }^{6,11,35,36}$ It is well known that addiction is associated with negative affectivity (eg, anxiety and depression) and that alcohol use in many cases can be considered as an avoidance coping strategy to escape from negative internal states and to reduce self-focused attention. ${ }^{10,24,37}$ Previous studies demonstrated the high co-occurrence of alcohol use disorders and depressive symptoms, and showed that depression and rumination negatively affected the treatment and prognosis of alcoholdependent patients. ${ }^{24,38,39}$ A review underlined that individuals with anxiety disorder, in particular panic disorder, had an increased risk of developing alcohol dependence. ${ }^{40}$

Psychological health and quality of life tend to improve after detoxification and treatment and worsen during relapse. ${ }^{35,36}$ Therefore, interventions focused on coping skills are important to train patients to use in everyday life alternative responses to triggers (eg, emotional distress) in place of drinking. ${ }^{8}$

The second aim of this study was to explore baseline predictors of the posttreatment temptation to drink.

Temptation to drink, usually known as craving, is a key area for addiction treatments since it is associated with increased risk of relapses and worse outcomes among substance dependence patients. ${ }^{23,41-43} \mathrm{We}$ found that posttreatment temptation to drink was significantly associated to polysubstance dependence and baseline readiness to change levels. Previous studies showed that polysubstance dependence is associated with negative outcomes since different drugs can enhance each other's negative effect with a synergistic result. ${ }^{44}$ Indeed, polysubstance abusers run the risk of developing an addiction to multiple substances and have an increased risk of developing serious physical and mental diseases. This is in line with a previous study among patients in a residential rehabilitation program, which showed that the polyabuser group scored higher in depression, anxiety, and psychological distress compared to the pure alcoholics group. ${ }^{6}$ Furthermore, a recent meta-analytic review of psychosocial interventions for substance use disorders found that treatments targeting polysubstance use were less effective and had the lowest percentage of posttreatment abstinence compared to other interventions for substance dependence. ${ }^{44}$ Therefore, polysubstance abuse interventions should be tailored to the individual's needs to ensure more effective and comprehensive treatment. ${ }^{45}$

Readiness to change is defined as a combination of the individual's perceived importance of the problem and their self-confidence in the ability to change. ${ }^{12}$ It has been widely used to measure motivation to change drinking behaviors and to suggest intervention strategies. ${ }^{16,17,32}$ Few studies have explored the association between readiness to change and craving; our results are in line with Chakravorty et al who found that readiness to change was an important predictor of craving among alcohol-dependent subjects, but additional studies are needed to clarify and to confirm this relationship. ${ }^{23}$

In our study, contrary to our hypothesis, baseline anxiety and depression were not significantly associated with posttreatment craving. This is not in line with previous literature showing that negative affectivity, both as a dispositional characteristic (eg, negative affectivity or neuroticism) and as a transient mood state, plays a key role in the craving experience. ${ }^{24,25,46}$ However, we found that patients with baseline medium-high craving had a significantly smaller reduction in their anxiety and depression levels during treatment compared to the group with low craving.

In this study, we also compared the psychological changes occurring during the 28-day rehabilitation program between patients with low versus medium-high temptation to drink at baseline. The longitudinal analysis performed showed that patients with low temptation to drink at baseline achieved a more significant improvement in motivation to change, self-efficacy, depression, and anxiety during rehabilitation 
compared to the group with medium-high levels. Thus, craving was negatively associated with mental health and treatment outcomes, as reported in previous studies. ${ }^{23}$ For example, Litt et al found that craving was related to drinking immediately following treatments and that it was most likely to occur in those who had severe dependence and mood disturbances. ${ }^{47}$

The findings should be evaluated in light of some study limitations. First, craving, anxiety, and depression were assessed in the context of a 28-day residential rehabilitation program for alcohol dependence in which subjects were committed to stop drinking; this could have led to a tendency to minimize self-reports. Therefore, results cannot be generalized to alcohol-dependent individuals in other social and cultural contexts. Second, the transtheoretical model of change is a pedagogical and conceptual framework but patients' readiness to change can fluctuate extremely quickly and change differently from individual to individual. Hence, readiness to change cannot be considered as a stable measure and in this study it would have been strongly affected by the rehabilitation treatment; therefore, caution is needed in interpreting the results and their predictive value.

Third, we evaluated temptation to drink using a self-report VAS that may not reflect the complex and multifactorial phenomenon of craving. Fourth, information about the presence of concurrent psychiatric disorders was not available because of the retrospective design of this study.

\section{Conclusion}

Our study showed that hospital rehabilitation for alcohol dependence problems is associated with an overall improvement in motivation to change and a significant reduction in psychological distress.

In addition, we found that baseline temptation to drink is negatively associated with mental health and treatment outcomes, and that the posttreatment temptation to drink was significantly associated with polysubstance dependence and baseline readiness to change levels.

These findings suggest the need for an early assessment of craving and careful monitoring during rehabilitation programs to better orient treatments so as to achieve positive outcomes; the temptation to drink assessment might be a useful tool for assessing the level of aftercare needs or whether an inpatient might need tailored treatment until the level of craving is reduced to prevent negative outcomes.

Interventions for alcohol-dependent patients should focus on motivation to change, negative moods, and relapse prevention strategies to help patients to identify high-risk situations and to manage craving more effectively. The time spent in treatment is one of the strongest factors associated with positive outcomes in the posttreatment period. ${ }^{48}$ Therefore, after a period of hospital rehabilitation, outpatient treatments based on an integrated care approach are fundamental to promote the retention of alcohol-dependent patients in active participation and to reduce the risk of relapses. ${ }^{49}$

Future research should focus on patient's assessment in the late detoxification period (ie, between the second and sixth months after discharge) to explore relationships among psychological changes, physical health, craving, and relapses.

\section{Acknowledgment}

The authors would like to express their appreciation to COordinamento Residenzialità Riabilitative ALcologiche (CORRAL).

\section{Disclosure}

The authors report no conflicts of interest in this work.

\section{References}

1. World Health Organization. Global Status Report on Alcohol and Health: 2014. Luxembourg: WHO; 2014.

2. Darvishi N, Farhadi M, Haghtalab T, Poorolajal J. Alcohol-related risk of suicidal ideation, suicide attempt, and completed suicide: a metaanalysis. PLoS One. 2015;10(5): 0126870.

3. Ronksley PE, Brien SE, Turner BJ, Mukamal KJ, Ghali WA. Association of alcohol consumption with selected cardiovascular disease outcomes: a systematic review and meta-analysis. BMJ. 2011;342:d671.

4. Testino G. Alcoholic diseases in hepato-gastroenterology: a point of view. Hepatogastroenterology. 2008;55(82-83):371-377.

5. National Institute on Alcohol Abuse and Alcoholism (NIAAA). Health risks and benefits of alcohol consumption. Alcohol Res Health. 2000;24(1):5-11.

6. Giorgi I, Ottonello M, Vittadini G, Bertolotti G. Psychological changes in alcohol-dependent patients during a residential rehabilitation program. Neuropsychiatr Dis Treat. 2015;11:2989.

7. Harris AH. A qualitative study on the introduction of mindfulness based relapse prevention (MBRP) into a therapeutic community for substance abusers. Int J Ther Commun. 2015;36(2):111-123.

8. Kadden R. Cognitive Behavioral Coping Skills Therapy Manual: A Clinical Research Guide for Therapists Treating Individuals with Alcohol Abuse and Dependence. Collingdale, PA: Diane Publishing; 1995.

9. Fiabane E, Ferraris E, Ranalletti P, et al. Dalla riabilitazione delle dipendenze alla reintegrazione al lavoro: Risultati di un progetto di reinserimento sociale e lavorativo per giovani con dipendenza da sostanze. [From the addiction rehabilitation program to the return to work: results of an employment and social intervention among young adults with substance dependence]. Med Lav. 2016;107(5):399-413. Italian.

10. Driessen M, Meier S, Hill A, Wetterling T, Lange W, Junghanns K. The course of anxiety, depression and drinking behaviours after completed detoxification in alcoholics with and without comorbid anxiety and depressive disorders. Alcohol Alcohol. 2001;36(3):249-255.

11. Lookatch SJ, Elledge LC, Anderson S, Shorey RC, Stuart GL, Moore TM. Cognitive and psychological changes during 28-day residential substance use treatment. Addict Res Theory. 2017;25(4):334-341. 
12. DiClemente CC, Schlundt D, Gemmell L. Readiness and stages of change in addiction treatment. Am J Addict. 2004;13:103-119.

13. Miller WR, Rollnick S. Motivational Interviewing: Helping People Change. 3rd ed. New York: Guilford Press; 2012.

14. Prochaska JO, DiClemente CC. Transtheoretical therapy: toward a more integrative model of change. Psychother Theory Res Pract. 1982; 19(3):276

15. DeLeon G, Melnick G, Kressel D. Motivation and readiness for therapeutic community treatment among cocaine and other drug abuses. Am J Drug Alcohol Abuse. 1997;23:169-189.

16. Gaume J, Bertholet N, Daeppen JB. Readiness to change predicts drinking: findings from 12-month follow-up of alcohol use disorder outpatients. Alcohol Alcohol. 2017;52(1):65-71.

17. Rumpf HJ, Hapke U, Meyer C, John U. Motivation to change drinking behaviour: comparison of alcohol-dependent individuals in a general hospital and a general population sample. Gen Hosp Psychiatry. 1999; 21:348-353.

18. Marlatt GA. Craving notes. Br J Addict. 1987;82:42-44.

19. Gordon SM, Sterling R, Siatkowski C, Raively K, Weinstein S, Hill PC. Inpatient desire to drink as a predictor of relapse to alcohol use following treatment. Am J Addict. 2006;15(3):242-245.

20. Schneekloth TD, Biernacka JM, Hall-Flavin DK, et al. Alcohol craving as a predictor of relapse. Am J Addict. 2001;21(1):S20-S26.

21. Witkiewitz K. Temptation to drink as a predictor of drinking outcomes following psychosocial treatment for alcohol dependence. Alcohol Clin Exp Res. 2013;37(3):529-537.

22. Higley AE, Crane NA, Spadoni AD, Quello SB, Goodell V, Mason BJ. Craving in response to stress induction in a human laboratory paradigm predicts treatment outcome in alcohol-dependent individuals. Psychopharmacology. 2011;218(1):121.

23. Chakravorty S, Kuna ST, Zaharakis N, O’Brien CP, Kampman KM, Oslin D. Covariates of craving in actively drinking alcoholics. Am J Addict. 2010;19(5):450-457.

24. Abulseoud OA, Karpyak VM, Schneekloth T, et al. A retrospective study of gender differences in depressive symptoms and risk of relapse in patients with alcohol dependence. Am J Addict. 2010;22(5):437-442.

25. Pombo S, Figueira ML, Walter H, Lesch O. Motivational factors and negative affectivity as predictors of alcohol craving. Psychiatry Res. 2016;243:53-60.

26. Witkiewitz K, Lustyk MKB, Bowen S. Retraining the addicted brain: a review of hypothesized neurobiological mechanisms of mindfulnessbased relapse prevention. Psychol Addict Behav. 2013;27(2):351.

27. American Psychiatric Association. Diagnostic and Statistical Manual of Mental Disorders. 4th ed (text revision). Washington: American Psychiatric Association; 2000.

28. Spiller V, Zavan V, Guelfi GP. Assessing motivation for change in subjects with alcohol problems: the MAC2-A questionnaire. Alcohol Alcohol. 2006;41(6):616-623.

29. Bertolotti G, Michielin P, Vidotto G, et al. Metric qualities of the cognitive behavioral assessment for outcome evaluation to estimate psychological treatment effects. Neuropsychiatr Dis Treat. 2015;11:2449.

30. Fukuoka Y, Dracup K, Takeshima M, et al. Effect of job strain and depressive symptoms upon returning to work after acute coronary syndrome. Soc Sci Med. 2009;68:1875-1881.

31. Fiabane E, Argentero P, Calsamiglia G, et al. Does job satisfaction predict early return to work after coronary angioplasty or cardiac surgery? Int Arch Occup Environ Health. 2013;86:561-569.
32. Cook S, Heather N, McCambridge J. Posttreatment motivation and alcohol treatment outcome 9 months later: findings from structural equation modeling. J Consult Clin Psychol. 2015;83(1):232-237.

33. Heather N, McCambridge J. Post-treatment stage of change predicts 12-month outcome of treatment for alcohol problems. Alcohol Alcohol. 2013;48:329-336.

34. Cadigan JM, Mertens MP, Arterberry BJ, Smith AE, Murphy JG. Examining a curvilinear model of readiness to change and alcohol consumption. Addict Res Theory. 2013;21(6):507-515.

35. Picci RL, Oliva F, Zuffranieri M, et al. Quality of life, alcohol detoxification and relapse: is quality of life a predictor of relapse or only a secondary outcome measure? Qual Life Res. 2014;23(10):2757-2767.

36. Frischknecht U, Sabo T, Mann K. Improved drinking behaviour improves quality of life: a follow-up in alcohol-dependent subjects 7 years after treatment. Alcohol Alcohol. 2013;48(5):579-584.

37. Spada MM, Zandvoort M, Wells A. Metacognitions in problem drinkers. Cognit Ther Res. 2007;31:709-716.

38. Caselli G, Bortolai C, Leoni M, Rovetto F, Spada MM. Rumination in problem drinkers. Addict Res Theory. 2008;16(6):564-571.

39. Zywiak WH, Stout RL, Trefry WB, et al. Alcohol relapse repetition, gender and predictive validity. J Subst Abuse Treat. 2006;30:349-353.

40. Brandizza CM, Stasiewicz PR, Paas ND. Relapse to alcohol and drug use among individuals diagnosed with co-occurring mental health and substance use disorders: a review. Clin Psychol Rev. 2006;26: $162-178$.

41. Bischof G, Rumpf HJ, Meyer C, Hapke U, John U. Gender differences in temptation to drink, self-efficacy to abstain and coping behavior in treated alcohol-dependent individuals: controlling for severity of dependence. Addict Res Theory. 2005;13(2):129-136.

42. Caselli G, Manfredi C, Ferraris A, Vinciullo F, Spada MM. Desire thinking as a mediator of the relationship between novelty seeking and craving. Addict Behav Rep. 2015;1:2-6.

43. Ray LA, Hutchison KE, Bryan A. Psychosocial predictors of treatment outcome, dropout, and change processes in a pharmacological clinical trial for alcohol dependence. Addict Disord Treat. 2006;5(4): 179-190.

44. Dutra L, Stathopoulou G, Basden SL, Leyro TM, Powers MB, Otto MW. A meta-analytic review of psychosocial interventions for substance use disorders. Am J Psychiatry. 2008;165(2):179-187.

45. Ogbu UC, Lotfipour S, Chakravarthy B. Polysubstance abuse: alcohol, opioids and benzodiazepines require coordinated engagement by society, patients, and physicians. West JEmerg Med. 2015;16(1):76-79.

46. Gamble SA, Conner KR, Talbot NL, Yu Q, Tu XM, Connors GJ. Effects of pretreatment and posttreatment depressive symptoms on alcohol consumption following treatment in Project MATCH. J Stud Alcohol Drugs. 2010;71(1):71-77

47. Litt MD, Cooney NL, Morse P. Reactivity to alcohol-related stimuli in the laboratory and in the field: predictors of craving in treated alcoholics. Addiction. 2000;95:889-900.

48. Elbreder MF, Silva RDS, Pillon SC, Laranjeira R. Alcohol dependence: analysis of factors associated with retention of patients in outpatient treatment. Alcohol Alcohol. 2011;46(1):74-76.

49. Litten RZ, Ryan ML, Falk DE, Reilly M, Fertig JB, Koob GF. Heterogeneity of alcohol use disorder: understanding mechanisms to advance personalized treatment. Alcohol Clin Exp Res. 2015;39(4):579-584.
Neuropsychiatric Disease and Treatment

\section{Publish your work in this journal}

Neuropsychiatric Disease and Treatment is an international, peerreviewed journal of clinical therapeutics and pharmacology focusing on concise rapid reporting of clinical or pre-clinical studies on a range of neuropsychiatric and neurological disorders. This journal is indexed on PubMed Central, the 'PsycINFO' database and CAS,

\section{Dovepress}

and is the official journal of The International Neuropsychiatric Association (INA). The manuscript management system is completely online and includes a very quick and fair peer-review system, which is all easy to use. Visit http://www.dovepress.com/testimonials.php to read real quotes from published authors. 